\title{
CYCLIC-PARALLEL REAL HYPERSURFACES OF QUATERNIONIC PROJECTIVE SPACE
}

\author{
By \\ Juan de Dios PEREZ
}

\begin{abstract}
We classify cyclic-parallel real hypersurfaces of quaternionic projective space.
\end{abstract}

\section{Introduction.}

Let $M$ be a connected real hypersurface of a quaternionic projective space $Q P^{m}, m \geqq 2$, endowed with the metric of constant quaternionic sectional curvature 4. If $\zeta$ denotes the unit local normal vector field and $\left\{J_{1}, J_{2}, J_{3}\right\}$ a local basis of the quaternionic structure of $Q P^{m}$ (see [2]), then $U_{i}=-J_{i} \zeta, i=1,2,3$, are vector fields tangent to $M$.

It is known, [4], that there do not exist parallel real hypersurfaces of $Q P^{m}, m \geqq 2$. A real hypersurface of $Q P^{m}$ is called cyclic-parallel if it satisfies

$$
\sigma\left(g\left(\left(\nabla_{X} A\right) Y, Z\right)\right)=0
$$

for any $X, Y, Z$ tangent to $M$, where $A$ denotes the Weingarten endomorphism of $M$ and $\sigma$ the cyclic sum.

Our purpose is to classify such real hypersurfaces by mean of the following

THEOREM. A real hypersurface $M$ of $Q P^{m}, m \geqq 2$, is cyclic-parallel if and only if it is congruent to an open subset of a tube of radius $r, 0<r<\Pi / 2$, over $Q P^{k}, k \in\{0, \cdots, m-1\}$.

In the Theorem, $Q P^{k}$ is considered canonically and totally geodesically embedded in $Q P^{m}$.

\section{Preliminaries.}

Let $X$ be a vector field tangent to $M$. We write $J_{i} X=\phi_{i} X+f_{i}(X) \zeta, i=1$, 2,3, where $\phi_{i} X$ denotes the tangent component of $J_{i} X$ and $f_{i}(X)=g\left(X, U_{i}\right)$. From this, see [3], we have

Received April 20, 1992.

Include: Research partially supported by DCICYT Grant PB90-0014-C03-02. 


$$
g\left(\phi_{i} X, Y\right)+g\left(X, \phi_{i} Y\right)=0, \quad \phi_{i} U_{i}=0, \quad \phi_{j} U_{k}=-\phi_{k} U_{j}=U_{t}
$$

for any $X, Y$ tangent to $M, i=1,2,3,(j, k, t)$ being a circular permutation of $(1,2,3)$.

From the expression of the curvature tensor of $Q P^{m}$ (see [2]) the equation of Codazzi is given by

$$
\left(\nabla_{X} A\right) Y-\left(\nabla_{Y} A\right) X=\sum_{i=1}^{3}\left\{f_{i}(X) \phi_{i} Y-f_{i}(Y) \phi_{i} X+2 g\left(X, \phi_{i} Y\right) U_{i}\right\}
$$

for any $X$ and $Y$ tangent to $M$. Moreover

$$
\nabla_{X} U_{i}=q_{k}(X) U_{j}-q_{j}(X) U_{k}+\phi_{i} A X
$$

for any $X$ tangent to $M,(i, j, k)$ being a circular permutation of $(1,2,3)$ and $q_{i}, i=1,2,3$, certain local 1 -forms on $M$ (see [2]).

In the following we shall denote by $\mathfrak{D}^{\perp}=\operatorname{Span}\left\{U_{1}, U_{2}, U_{s}\right\}$ and by $\mathscr{O}$ its orthogonal complement in $T M$.

\section{Proof of Theorem.}

From (1.1) and applying twice (2.2) we find

$$
\left(\nabla_{X} A\right) Y=-\sum_{i=1}^{3}\left\{f_{i}(Y) \phi_{i} X+g\left(Y, \phi_{i} X\right) U_{i}\right\} .
$$

From (3.1), (2.1) and (2.3) we have for any $X, Y, Z$ tangent to $M$

$$
\begin{aligned}
\left(\nabla_{Z}\left(\nabla_{X} A\right)\right) Y-\left(\nabla_{\nabla_{Z} X} A\right) Y= & -\sum_{i=1}^{3}\left\{g\left(\phi_{i} X, Y\right) \phi_{i} A Z\right. \\
& +g\left(Y, \phi_{i} A Z\right) \phi_{i} X+f_{i}(X) g(A Z, Y) U_{i} \\
& \left.-2 f_{i}(Y) g(A X, Z) U_{i}+f_{i}(Y) f_{i}(X) A Z\right\} .
\end{aligned}
$$

If we exchange $Z$ and $X$, from (3.2) we obtain

$$
\begin{aligned}
(R(Z, X) A) Y= & -\sum_{i=1}^{3}\left\{f_{i}(X) g(A Z, Y) U_{i}-f_{i}(Z) g(A X, Y) U_{i}\right. \\
& +g\left(\phi_{i} X, Y\right) \phi_{i} A Z-g\left(\phi_{i} Z, Y\right) \phi_{i} A X+g\left(\phi_{i} A Z, Y\right) \phi_{i} X \\
& \left.-g\left(\phi_{i} A X, Y\right) \phi_{i} Z+f_{i}(Y) f_{i}(X) A Z-f_{i}(Y) f_{i}(Z) A X\right\} .
\end{aligned}
$$

Let now $\left\{E_{j}, j=1, \cdots, 4 m-1\right\}$ be an orthonormal frame on $M$. From (3.3) we have

$$
\begin{aligned}
\sum_{j=1}^{4 m-1} g\left(\left(R\left(E_{j}, X\right) A\right) Y, E_{j}\right)= & -\sum_{i=1}^{3}\left\{f_{i}(X) f_{i}(A Y)-g\left(\phi_{i} X, Y\right) \operatorname{trace}\left(A \phi_{i}\right)\right. \\
& -2 f_{i}(Y) f_{i}(A X)-g\left(A \phi_{i} Y, \phi_{i} X\right) \\
& \left.+\operatorname{trace}(A) f_{i}(Y) f_{i}(X)\right\}
\end{aligned}
$$

But the left hand side of (3.4) is symmetric respect to $X$ and $Y$. This gives 


$$
-3 \sum_{i=1}^{3} f_{i}(X) f_{i}(A Y)=-3 \sum_{i=1}^{3} f_{i}(Y) f_{i}(A X)+2 \sum_{i=1}^{3} g\left(\phi_{i} Y, X\right) \operatorname{trace}\left(A \phi_{i}\right) .
$$

From $(2.1)$ it is easy to see that trace $\left(A \phi_{i}\right)=0$. This implies

$$
\sum_{i=1}^{3} f_{i}(X) f_{i}(A Y)=\sum_{i=1}^{3} f_{i}(Y) f_{i}(A X)
$$

for any $X, Y$ tangent to $M$. From (3.6) we obtain that $g\left(A \mathscr{D}, \mathfrak{D}^{\perp}\right)=\{0\}$. Thus, [1], $M$ is congruent to an open subset of either a tube of radius $r, 0<r<\Pi / 2$, over $Q P^{k} \quad k \in\{0, \cdots, m-1\}$ or a tube of radius $r, 0<r<\Pi / 4$ over $C P^{m}$.

If we consider the second case, the principal curvatures of $M$ are, [3], $\cot (r),-\tan (r), 2 \cot (2 r),-2 \tan (2 r)$ with respective multiplicities $2(m-1)$, $2(m-1), 1$ and 2 . If $X$ is a unit vector field such that $A X=-\tan (r) X$, from (2.3) the $\mathfrak{D}$-component of $\left(\nabla_{X} A\right) U_{2}$ is $-\tan (r)(-2 \tan (2 r)-\cot (r)) \phi_{2} X$. But if $M$ is cyclic-parallel from (3.1) $\left(\nabla_{X} A\right) U_{2}$ must be equal to $-\phi_{2} X$. This implies that $\cot ^{2}(r)=-1$ which is impossible.

On the other hand it is easy to prove that the tubes over $Q P^{k}$ are cyclicparallel and this finishes the proof.

\title{
References
}

[1] J. Berndt, Real hypersurfaces in quaternionic space forms, J. reine angew. Math., 419 (1991), 9-26.

[2] S. Ishihara, Quaternion Kählerian manifolds, J. Differential Geom., 9 (1974), 483500 .

[3] A. Martínez and J.D. Pérez, Real hypersurfaces in quaternionic projective space, Ann. di Mat., 145 (1986), 355-384.

[4] J.S. Pak, Real hypersurfaces in quaternionic Kaehlerian manifolds with constant $Q$-sectional curvature, Kodai Math. Sem. Rep., 29 (1977), 22-61.

\author{
Departmento de Geometría y Topología \\ Facultad de Ciencias \\ Universidad de Granada \\ 18071-GRANADA, SPAIN
}

